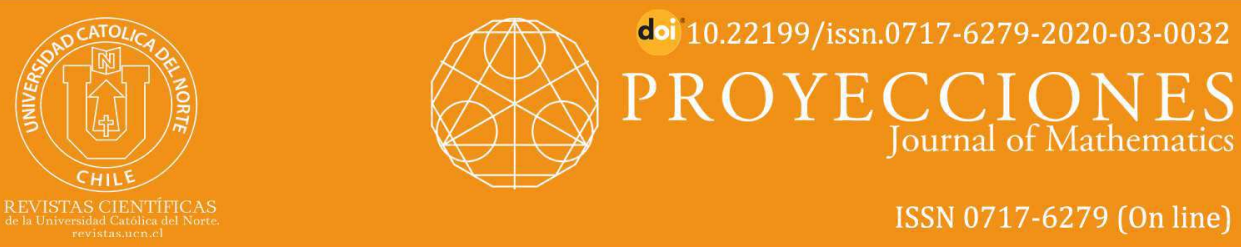

\title{
Total graph of a commutative semiring with respect to singular ideal
}

Nabanita Goswami ${ }^{1}$ (C) orcid.org/0000-0003-3395-6300

Helen K. Saikia ${ }^{2}$ (1) orcid.org/0000-0003-1971-9472

Gauhati University, Dept. of Mathematics, Guwahati, AS, India.

1ఐnabanita01goswami@gmail.com ; ${ }^{2 \varpi}$ hsaikia@yahoo.com

\section{Abstract:}

Let $S$ be a commutative semiring with unity. The singular ideal $Z(S)$ of $S$ is defined as $Z(S)=\{s \in S / s K=0$ for some essential ideal $K$ of $S\}$. In this paper, we introduce the notion of total graph of a commutative semiring with respect to the singular ideal. We define this graph as the undirected graph $T(\Gamma(S))$ with all elements of $S$ as vertices and any two distinct vertices $x$ and $y$ are adjacent if and only if $x+y \in Z(S)$. We discuss various characteristics of this total graph and also characterize some important properties of certain induced subgraphs of this total graph.

Keywords: Semiring; Total graph; Singular ideal; Induced Subgraphs.

MSC (2010): 05C25, 05C75, 16Y60.

\section{Cite this article as (IEEE citation style)}

N. Goswami and H. K. Saikia, "Total graph of a commutative se-

miring with respect to singular ideal", Proyecciones (Antofagasta, On line), vol. 39, no. 3, pp. 517-527, Jun. 2020, doi: 10.22199/issn.0717-6279-2020-03-0032.

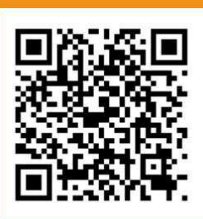

Article copyright: (c) 2020 Nabanita Goswami and Helen K. Saikia. This is an open access article distributed under the terms of the Creative Commons Licence, which permits unrestricted use and distribution provided the original author and source are credited

(cc) BY 


\section{Introduction}

In the last two decades, the study of algebraic structures using the properties of graphs has emerged as an exciting area of research. This study began way back in 1988 with Istvan Beck establishing a correspondence between the theory of graph and ring theory. In his paper [18], Beck introduced the notion of coloring of a commutative ring by defining the zero-divisor graph of a commutative ring. The various properties of the graph defined by I. Beck is extensively studied by different authors $[2,3,4,7,8]$ in the following years. Motivated by the idea of zero divisor graph, researchers have associated graphs with different algebraic structures in various ways. Some of them are regular graph of a ring, total graph of a ring, unit graph of a ring, the inclusion ideal graph of a ring, the nil-graph of ideals of commutative ring and co-maximal graph of a ring etc. In the study of the graphs constructed out of different algebraic structures like rings, modules etc, researchers mostly study the interplay between the algebraic properties and the graph theoretical properties of the graphs.

Among the graphs mentioned above, the concept of total graph of commutative ring was introduced by Anderson et al.[6] in 2008. For a commutative ring $R$, they defined this graph as the undirected graph with $R$ as the vertex set and if $x$ and $y$ are any two vertices then they are adjacent if and only if $x+y \in Z(R)$, where $Z(R)$ denotes the set of zero divisors of $R$. Different properties of this graph are investigated by many authors $[9,10]$ and also the study of several other variants of the total graph are found in the literature $[1,5,10,13,21,22]$. The total graph defined on various algebraic structures involves both the operations associated with the structure instead of only multiplication, which is the case for zero-divisor graph and so the study of total graphs of various algebraic structures reveal more of the important properties than the study of zero-divisor graph. In [21] the authors have introduced the notion of total graph of a module with respect to singular submodule. For a commutative ring $R$ with unity and an $R$-module $M$ they define the total graph of $M$ with respect to singular submodule $Z(M)$ of $M$ as an undirected graph with vertex set $M$ and any two distinct vertices $x$ and $y$ are adjacent if and only if $x+y \in Z(M)$.

The theory of semiring was first introduced by H. S. Vandiver in 1934 [24]. In recent development of theory, semiring has achieved an importance as it has got many applications in different areas like combinatorics and graph theory, Euclidean geometry and topology, functional analysis, automata and formal language, mathematical modeling of quantum physics, 
probability theory etc. Various graphs related to semirings have been studied recently. Also, the notion of various graphs like zero-divisor graphs and total graphs related to ring and module has been extended to semiring and this area of research is also growing rapidly. S.E. Atani has studied the zero divisor graph and total graph of commutative semiring in his papers $[11,12]$ and some other variants of total graph of semiring are investigated by many authors $[14,15,16,17,23]$.

In this paper we define the total graph of a commutative semiring $S$ with respect to the singular ideal $Z(S)$. This graph is defined as the undirected graph $T(\Gamma(S))$ with $S$ as the vertex set and two vertices $x$ and $y(x \neq y)$ are adjacent if $x+y \in Z(S)$. We characterize this graph and also study the interplay between algebraic properties of $S$ and graph theoretic properties of $T(\Gamma(S))$. For any subset $A$ of $S$, the induced subgraph of $T(\Gamma(S))$ with all elements of $A$ as vertices is denoted by $T(\Gamma(A))$. We discuss some characteristics of two induced subgraphs $T(\Gamma(Z(S)))$ and $T(\Gamma(\overline{Z(S)}))$ of this graph where $\overline{Z(S)}=S \backslash Z(S)$.

\section{Preliminaries}

In this section, we present some basic definitions and notations which will be used in the subsequent sections. General references for graph theory and semiring theory are [19] and [20] respectively.

Let $G$ be a simple graph. $G$ is said to be connected if every pair of distinct vertices are connected by a path. If no two vertices of $G$ are adjacent then $G$ is called totally disconnected. The graph $G$ is called a complete graph if every pair of distinct vertices are adjacent. A complete graph with $n$ vertices is denoted by $K_{n}$. The graph $G$ is said to be acyclic if it has no cycles. If $G$ is a connected acyclic graph then it is called a tree. $G$ is called a star graph if it is a tree of order $n$ with maximum diameter 2. For any two distinct vertices $x$ and $y$ of $G$, the distance between $x$ and $y$ is the length of a shortest path connecting $x$ and $y$ which is denoted by $d(x, y) \cdot d(x, y)=\infty$ if no such path exists. The diameter $\operatorname{diam}(G)$ of $G$ is defined as $\operatorname{diam}(G)=\sup \{d(x, y) \mid x$ and $y$ are vertices of $G\}$. The girth, $\operatorname{gr}(G)$ of $G$ is the length of a shortest cycle in $G$, if $G$ contains a cycle; otherwise $\operatorname{gr}(G)=\infty$.

A graph $H$ is said to be an induced subgraph of $G$ if the vertex set of $H$ is a subset of the vertex set of $G$ and two vertices are adjacent in $H$ if and only if they are adjacent in $G$. Two (induced) subgraphs $H_{1}$ and $H_{2}$ of $G$ are said to be disjoint if the vertices of $H_{1}$ are different from the vertices of 
$H_{2}$ and no vertex of $H_{1}$ (respectively, $H_{2}$ ) is adjacent (in $G$ ) to any vertex not in $H_{1}$ (respectively, $H_{2}$ ).

A semiring is a non-empty set $S$ together with two binary operations + and $\cdot$ such that $(S,+)$ is a commutative monoid with identity element 0 and $(S, \cdot)$ is a monoid with identity 1 , furthermore multiplication distributes over addition from either side and 0 is multiplicatively absorbing. A semiring $S$ is said to be a commutative if $(S, \cdot)$ is a commutative monoid.

An ideal of a commutative semiring $S$ is a non-empty subset $I$ of $S$ such that $a+b \in I$ and $a s \in I$ for $a, b \in I, s \in S$. An ideal $I$ of a semiring $S$ is called a $k$-ideal (subtractive ideal) if and only if $x \in I$ and $x+y \in I$ imply $y \in I$. An ideal $I$ of a semiring $S$ is said to be an essential ideal if $I \cap K \neq 0$ for every nonzero ideal $K$ of $S$.

The singular ideal $Z(S)$ of a commutative semiring $S$ is defined as $Z(S)=\{s \in S \mid s K=0$ for some essential ideal $K$ of $S\} . \overline{Z(S)}$ denotes the complement of $Z(S)$, i.e. $\overline{Z(S)}=S \backslash Z(S)$.

\section{On the graph $T(\Gamma(S))$ of a commutative semiring $S$}

Throughout this section $S$ represents a commutative semiring with nonzero identity and $Z(S)$ denotes the singular ideal of $S$. In this section, we discuss the characteristics of this total graph $T(\Gamma(S))$ and also characterize some important properties of the two induced subgraphs $T(\Gamma(Z(S))$ and $T(\Gamma(\overline{Z(S)})$ of this graph.

Proposition 3.1. The singular ideal $Z(S)$ of a commutative semiring $S$ is a $k$-ideal.

Proof. Let $x \in Z(S)$ and $x+y \in Z(S)$ for some $x, y \in S$. As $Z(S)$ is the singular ideal, there exist essential ideals $I, J$ of $S$ such that $x I=0$ and $(x+y) J=0$. Since the intersection of two essential ideals of a semiring is again an essential ideal, we have $I \cap J$ is essential in $S$. Let $K=I \cap J$, then obviously $x K=0$ and $(x+y) K=0$. From this we get $y K=0$ and this implies that $y \in Z(S)$. Hence $Z(S)$ is a $k$-ideal of $S$.

Theorem 3.2. For $s \in \overline{Z(S)}, 2 \in Z(S)$ if and only if $2 s \in Z(S)$.

Proof. Let $2 \in Z(S)$ and $s \in \overline{Z(S)}$, then as $Z(S)$ is an ideal of $S$ so $2 s \in Z(S)$. 
Conversely, we suppose that $2 s \in Z(S)$ for $s \in \overline{Z(S)}$. Now, $1 \notin Z(S)$ for if $1 \in Z(S)$ then $1 . I=0$ for some essential ideal $I$ of $S$ which implies that 0 is essential in $S$ and this is not possible. So we have $1 \in \overline{Z(S)}$ and hence $2 \in Z(S)$.

Theorem 3.3. The induced subgraph $T(\Gamma(Z(S))$ of $T(\Gamma(S))$ is complete and $T(\Gamma(Z(S))$ is disjoint from $T(\Gamma(\overline{Z(S)})$.

Proof. 1st part of the theorem follows directly from the definition.

2nd Part:

If possible we suppose that the graphs $T(\Gamma(Z(S))$ and $T(\Gamma(\overline{Z(S)})$ are not disjoint. Then we must have $s_{1}+s_{2} \in Z(S)$ for some $s_{1} \in Z(S)$ and $s_{2} \in \overline{Z(S)}$. As $Z(S)$ is a $k$-ideal we get $s_{2} \in Z(S)$ which is a contradiction. So our supposition is wrong. Hence $T(\Gamma(Z(S))$ is disjoint from $T(\Gamma(\overline{Z(S)})$.

Example 3.1. Let $S=\{0, x, 1, a\}$ where $\{0, x, 1\}$ is a linearly ordered lattice with $0 \leq x \leq 1$. Also $a^{2}=0,2 a=a, x a=a, x+a=x$ and $1+a=1$. Then $S$ forms a semiring.

The singular ideal of $S$ is $Z(S)=\{0, a\}$. The graph $T(\Gamma(S))$ is as given in figure 1.

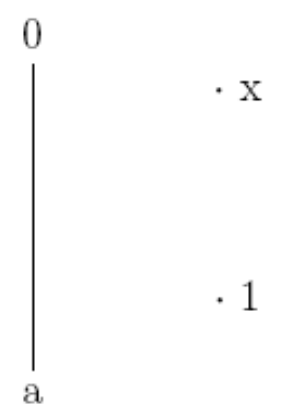

Figure 1: $T(\Gamma(S))$

In the example 3.1., we can see that $T(\Gamma(Z(S)))$ is a complete induced subgraph of $T(\Gamma(S))$ and $T(\Gamma(Z(S)))$ is disjoint from $T(\Gamma(\overline{Z(S)}))$. 
Remark: Theorem 3.3. gives us a complete description of the induced subgraph $T(\Gamma(Z(S))$ of $T(\Gamma(S))$ and so in the next part of this section we discuss the induced subgraph $T(\Gamma(\overline{Z(S)})$ of $T(\Gamma(S))$.

Theorem 3.4. Let $s_{1}, s_{2}\left(s_{1} \neq s_{2}\right)$ are vertices of $T(\Gamma(\overline{Z(S)})$ such that they are connected by a path. If $s_{1}$ and $s_{2}$ are not adjacent, then between $s_{1}$ and $s_{2}$ there is a path in the graph $T(\Gamma(\overline{Z(S)})$ of length at most 2. In particular, if $T(\Gamma(\overline{Z(S)}))$ is connected, then $\operatorname{diam}(T(\Gamma(\overline{Z(S)}))) \leq 2$.

Proof. Let us consider the vertices $r, s, t, u$ of $T(\Gamma(\overline{Z(S)})$ such that they are distinct. It serves our purpose if we can show that if there is a path $r-s-t-u$ from $r$ to $u$, then the vertices $r$ to $u$ are adjacent. Since there is a path $r-s-t-u$ from $r$ to $u$ in $T(\Gamma(\overline{Z(S)})$, so $r+s, s+t, t+u \in Z(S)$. This implies $r+s+t+u \in Z(S)$ as $Z(S)$ is an ideal. Again as $Z(S)$ is a $k$-ideal of $S$ we have $r+u \in Z(S)$. Hence $r$ and $u$ are adjacent, as required. Again if $T(\Gamma(\overline{Z(S)}))$ is connected, then clearly diam $(T(\Gamma(\overline{Z(S)}))) \leq 2$.

Theorem 3.5. The following hold:

(i) If $2 \in Z(S)$, then $T(\Gamma(\overline{Z(S)})$ is the union of disjoint complete subgraphs.

(ii) If $2 \notin Z(S)$, then $T(\Gamma(\overline{Z(S)})$ is the union of totally disconnected subgraphs and some connected subgraphs.

(i) Here $2 \in Z(S)$. Let $s \in \overline{Z(S)}$. We consider the set $s+Z(S)$. As $Z(S)$ is a $k$-ideal of $S$ so for any $a \in Z(S), s+a \in \overline{Z(S)}$ and hence $s+Z(S) \subseteq \overline{Z(S)}$. Now as $2 \in Z(S)$ so by Theorem 3.2., for $s \in \overline{Z(S)}$, $2 s \in Z(S)$. We take $a, b \in Z(S)$. Then $s+a, s+b \in s+Z(S)$ and $(s+a)+(s+b)=2 s+(a+b) \in Z(S)$. This implies that the subgraph $T(\Gamma((s+Z(S))))$ of $T(\Gamma(\overline{Z(S)}))$ with vertex set $s+Z(S)$ is a complete subgraph. Now if for any two distinct $r, s \in \overline{Z(S)}, r+Z(S)$ and $s+Z(S)$ are disjoint then clearly the induced subgraphs with the vertex sets $r+Z(S)$ and $s+Z(S)$ are also disjoint. If for some $r, s \in \overline{Z(S)}, r+Z(S)$ and $s+Z(S)$ are not disjoint, then we get $r+a \in r+Z(S)$ and $s+b \in s+Z(S)$ such that $(r+a)+(s+b) \in Z(S)$ for some $a, b \in Z(S)$. Then $r+s \in Z(S)$ since $Z(S)$ is a $k$-ideal. This implies that for any elements $r+z_{1} \in r+Z(S)$ and $s+z_{2} \in s+Z(S),\left(r+z_{1}\right)+\left(s+z_{2}\right) \in Z(S)$, i.e. each vertex of $r+Z(S)$ is adjacent to each vertex of $s+Z(S)$ and so the induced subgraph of $T(\Gamma(\overline{Z(S)}))$ with vertex set $(r+Z(S)) \cup(s+Z(S))$ is complete. Thus we get that the graph $T(\Gamma(\overline{Z(S)}))$ is the union of disjoint complete 
subgraphs.

(ii) Here $2 \notin Z(S)$. We consider an element $s \in \overline{Z(S)}$. Now we show that the induced subgraph with vertex set $s+Z(S)$ of the graph $T(\Gamma(\overline{Z(S)}))$ is totally disconnected. If possible we suppose that this induced subgraph is not totally disconnected. Then there exist $s+a, s+b \in s+Z(S)$ such that they are adjacent. Which implies $(s+a)+(s+b) \in Z(S)$ i.e. $2 s+a+b \in Z(S)$ and since $Z(S)$ is a $k$-ideal we have $2 s \in Z(S)$. But this is a contradiction to the fact that $2 \notin Z(S)$ as by Theorem 3.2, we have for $s \in \overline{Z(S)}$, $2 s \in Z(S)$ implies that $2 \in Z(S)$. Thus we have, that the induced subgraph of $T(\Gamma(\overline{Z(S)}))$ with vertex set $s+Z(S)$ is totally disconnected. Now if for any two distinct $r, s \in \overline{Z(S)}$, the induced subgraphs with the vertex sets $r+Z(S)$ and $s+Z(S)$ are also disjoint then there is nothing to prove. If for some $r, s \in \overline{Z(S)}, r+Z(S)$ and $s+Z(S)$ are not disjoint, then there exist $r+a \in r+Z(S)$ and $s+b \in s+Z(S)$ for some $a, b \in Z(S)$ such that $(r+a)+(s+b) \in Z(S)$. This implies $r+s \in Z(S)$ as $Z(S)$ is a $k$-ideal. From this we get that for any elements $r+z_{1} \in r+Z(S)$ and $s+z_{2} \in s+Z(S)$, $\left(r+z_{1}\right)+\left(s+z_{2}\right) \in Z(S)$, i.e. each element of $T(\Gamma(r+Z(S)))$ is adjacent to each element of $T(\Gamma(s+Z(S)))$ and so the induced subgraph of $T(\Gamma(\overline{Z(S)}))$ with vertex set $(r+Z(S)) \cup(s+Z(S))$ is connected. Hence we have, if $2 \notin Z(S)$, then $T(\Gamma(\overline{Z(S)}))$ is the union of totally disconnected subgraphs and some connected subgraphs.

Theorem 3.6. Let $2 \notin Z(S)$. If the induced subgraph $T(\Gamma(\overline{Z(S)})$ of $T(\Gamma(S))$ is complete, then either $|\overline{Z(S)}|=1$ or 2 .

Proof. Here $2 \notin Z(S)$. Let us consider an element $s \in \overline{Z(S)}$. Then we have shown in the proof of the Theorem 3.5. that the induced subgraph $T(\Gamma(s+Z(S)))$ of $T(\Gamma(\overline{Z(S)}))$ is totally disconnected. Also we have shown that if for any two distinct $r, s \in \overline{Z(S)}$, the induced subgraphs with the vertex sets $r+Z(S)$ and $s+Z(S)$ are not disjoint then each element of $T(\Gamma(r+Z(S)))$ is adjacent to each element of $T(\Gamma(s+Z(S)))$. Now as the graph $T(\Gamma(\overline{Z(S)}))$ is complete, so from the first case we have $T(\Gamma(\overline{Z(S)}))$ is the complete graph $K_{1}$ with one vertex. Thus $|\overline{Z(S)}|=1$. Again in the second case $T(\Gamma(\overline{Z(S)}))$ is the complete graph with two vertex and so $|\overline{Z(S)}|=2$. Thus we have if $2 \notin Z(S)$ and $T(\Gamma(\overline{Z(S)}))$ is complete, then either $|\overline{Z(S)}|=1$ or 2 . 


\section{Theorem 3.7.}

(i) If $2 \in Z(S)$, then $\operatorname{diam}(T(\Gamma(\overline{Z(S)})))=0,1$ or $\infty$.

(ii) If $2 \notin Z(S)$, then $\operatorname{diam}(T(\Gamma(\overline{Z(S)})))=0,1,2$ or $\infty$.

(i) We take $2 \in Z(S)$. From Theorem 3.4. we have if $T(\Gamma(\overline{Z(S)}))$ is connected then $\operatorname{diam}(T(\Gamma(\overline{Z(S)}))) \leq 2$. Again by Theorem 3.5.(i) we have $T(\Gamma(\overline{Z(S)}))$ is the union of disjoint complete subgraphs and so $\operatorname{diam}(T(\Gamma(\overline{Z(S)}))) \neq 2$. Hence $\operatorname{diam}(T(\Gamma(\overline{Z(S)})))=0,1$ or $\infty$.

(ii) Obvious from Theorem 3.4.

Theorem 3.8. $\operatorname{diam}(T(\Gamma(\overline{Z(S)}))=0$ if and only if $|\overline{Z(S)}|=1$.

Proof. $\quad \operatorname{Let} \operatorname{diam}(T(\Gamma(\overline{Z(S)}))=0$. Then clearly $T(\Gamma(\overline{Z(S)})$ is the complete graph $K_{1}$ with one vertex, and so $|\overline{Z(S)}|=1$. Converse part is trivial.

Theorem 3.9. $\operatorname{gr}(T(\Gamma(\overline{Z(S)})) \leq 4$ if $T(\Gamma(\overline{Z(S)})$ contains a cycle. Otherwise $\operatorname{gr}(T(\Gamma(\overline{Z(S)}))=\infty$.

Proof. We suppose that $T(\Gamma(\overline{Z(S)})$ contains a cycle. Then it is not possible that $T(\Gamma(\overline{Z(S)})$ is totally disconnected. Now by the proof of Theorem 3.5., $T(\Gamma \overline{Z(S)})$ has either a complete subgraph or a complete bipartite subgraph, which means it either contains a 3-cycle or contains a 4-cycle and hence $\operatorname{gr}(T(\Gamma(\overline{Z(S)})) \leq 4$.

Theorem 3.10. The following hold:

(i) $\operatorname{gr}(T(\Gamma(\overline{Z(S)}))=3$ if and only if $2 \in Z(S)$ and $|s+Z(S)| \geq 3$ for some $s \in \overline{Z(S)}$.

(ii) $\operatorname{gr}\left(T(\Gamma(\overline{Z(S)}))=4\right.$ if and only if $2 \notin Z(S), s_{1}+s_{2} \in Z(S)$ and $\mid s_{1}+$ $Z(S)|,| s_{2}+Z(S) \mid \geq 2$ for some $s_{1}, s_{2} \in \overline{Z(S)}$.

\section{Proof.}

(i) If $\operatorname{gr}(T(\Gamma(\overline{Z(S)}))=3$, then by Theorem 3.5. $T(\Gamma(\overline{Z(S)})$ is a complete graph $K_{\alpha}$ with $\alpha \leq 3$. Therefore, $2 \in Z(S)$ and $|s+Z(S)| \geq 3$ for some $s \in \overline{Z(S)}$. The converse part of (i) is obvious from Theorem 3.5. 
(ii) If $\operatorname{gr}(T(\Gamma(\overline{Z(S)}))=4$, then by Theorem 3.5., $T(\Gamma(\overline{Z(S)})$ has a complete bipartite subgraph; which implies $2 \notin Z(S)$ and $s_{1}+s_{2} \in Z(S)$ for some $s_{1}, s_{2} \in \overline{Z(S)}$. Also it is clear that $\left|s_{1}+Z(S)\right|,\left|s_{2}+Z(S)\right| \geq 2$. Conversely, from Theorem 3.5., the graph with vertex set $\left(s_{1}+Z(S)\right) \cup\left(s_{2}+Z(S)\right)$ is a complete bipartite subgraph of $T\left(\Gamma(\overline{Z(S)})\right.$. Again, $\left|s_{1}+Z(S)\right|,\left|s_{2}+Z(S)\right| \geq$ 2 implies that $\operatorname{gr}(T(\Gamma(\overline{Z(S)}))=4$.

Theorem 3.11. The following hold:

(i) $\operatorname{gr}(T(\Gamma(S)))=3$ if and only if $|Z(S)| \geq 3$.

(ii) $\operatorname{gr}(T(\Gamma(S)))=4$ if and only if $2 \notin Z(S),|Z(S)|<3$ and $s_{1}+s_{2} \in Z(S)$, $\left|s_{1}+Z(S)\right|,\left|s_{2}+Z(S)\right| \geq 2$ for some $s_{1}, s_{2} \in \overline{Z(S)}$.

(iii) Otherwise, $\operatorname{gr}(T(\Gamma(S)))=\infty$.

\section{Proof.}

(i) We suppose that $\operatorname{gr}(T(\Gamma(S)))=3$. We know that $T(\Gamma(Z(S)))$ is disjoint from $T(\Gamma(\overline{Z(S)}))$ which means that either $\operatorname{gr}(T(\Gamma(Z(S))))=3$ or $\operatorname{gr}(T(\Gamma(\overline{Z(S)})))=3$. If $\operatorname{gr}(T(\Gamma(Z(S))))=3$ then clearly $|Z(S)| \geq 3$. Again if $\operatorname{gr}(T(\Gamma(\overline{Z(S)})))=3$, then by Theorem 3.10, $|s+Z(S)| \geq 3$ for some $s \in Z(S)$ which implies that $|Z(S)| \geq 3$. Conversely, $|Z(S)| \geq 3$ implies that $\operatorname{gr}(T(\Gamma(Z(S))))=3$ and since $T(\Gamma(Z(S)))$ is a complete induced subgraph of $T(\Gamma(S))$, so $\operatorname{gr}(T(\Gamma(S)))=3$.

(ii) Let $\operatorname{gr}(T(\Gamma(S)))=4$. Since the induced subgraph $T(\Gamma(Z(S)))$ of $T(\Gamma(S))$ is complete, so $\operatorname{gr}(T(\Gamma(Z(S)))=3$ or 1 and hence we must have $\operatorname{gr}(T(\Gamma(\overline{Z(S)})))=$ 4. Therefore by Theorem 3.10, we have $2 \notin Z(S), s_{1}+s_{2} \in Z(S)$ and $\left|s_{1}+Z(S)\right|,\left|s_{2}+Z(S)\right| \geq 2$ for some $s_{1}, s_{2} \in \overline{Z(S)}$. Again $\operatorname{gr}(T(\Gamma(S))) \neq$ 3 implies that $|Z(S)|<3$. Conversely, using Theorem 3.10, we have $\operatorname{gr}(T(\Gamma(\overline{Z(S)})))=4$. Again $|Z(S)|<3$ implies that $\operatorname{gr}(T(\Gamma(Z(S))))=\infty$ i.e. $\operatorname{gr}(T(\Gamma(Z(S))))$ can not be equal to 3 . Hence $\operatorname{gr}(T(\Gamma(S)))=4$.

(iii) Obvious from definition.

\section{Acknowledgment}

The first author would like to thank the Department of Science and Technology, Government of India, for their financial support. 


\section{References}

[1] A. Abbasi and S. Habibi, "The total graph of a commutative ring with respect to proper ideals", Journal of the Korean Mathematical Society, vol. 49, no. 1, pp. 85-98, Jan. 2012, doi: 10.4134/JKMS.2012.49.1.085

[2] S. Akbari and A. Mohammadian, "On the zero-divisor graph of a commutative ring", Journal of algebra, vol. 274, no. 2, pp. 847-855, Apr. 2004, doi: 10.1016/S0021-8693(03)00435-6

[3] D. F. Anderson and M. Naseer, "Beck's coloring of a commutative ring", Journal of algebra, vol. 159, no. 2, pp. 500-514, Aug. 1993, doi: 10.1006/jabr.1993.1171

[4] D. F. Anderson, "On the diameter and girth of a zero-divisor graph, II", Houston journal of mathematics, vol. 34, no. 2, pp. 361-371, 2008.

[5] D. F. Anderson and A. Badawi, "The generalized total graph of a commutative ring", Journal of algebra and its applications, vol. 12, no. 05, pp. 125-212, May 2013, doi: 10.1142/S021949881250212X

[6] D. F. Anderson and A. Badawi, "The total graph of a commutative ring", Journal of algebra, vol. 320, no. 7, pp. 2706-2719, Oct. 2008, doi: 10.1016/j.jalgebra.2008.06.028

[7] D. F. Anderson and P. S. Livingston, "The zero-divisor graph of a commutative ring", Journal of algebra, vol. 217, no. 2, pp. 434-447, Jul. 1999, doi: 10.1006/jabr.1998.7840

[8] D. F. Anderson and S. Mulay, "On the diameter and girth of a zerodivisor graph", Journal of pure and applied algebra, vol. 210, no. 2, pp. 543-550, Aug. 2007, doi: 10.1016/j.jpaa.2006.10.007

[9] T. Asir and T. Tamizh Chelvam, "Genus of total graphs of commutative rings: a survey", Electronic notes in discrete mathematics, vol. 63, pp. 59-68, Dec. 2017, doi: 10.1016/j.endm.2017.10.062

[10] T. Asir and T. T. Chelvam, "On the total graph and its complement of a commutative ring", Communications in algebra, vol. 41, no. 10, pp. 3820-3835, Oct. 2013, doi: 10.1080/00927872.2012.678956

[11] S. E. Atani, "An ideal based zero-divisor graph of a commutative semiring", Glasnik matematicki, vol. 44, no. 1, pp. 141-153, May 2009, doi: 10.3336/gm.44.1.07

[12] S. E. Atani, "The zero-divisor graph with respect to ideals of a commutative semiring", Glasnik matematicki, vol. 43, no. 2, pp. 309-320, Nov. 2008, doi: 10.3336/gm.43.2.06

[13] S. E. Atani and S. Habibi, "The total torsion element graph of a module over a commutative ring", Analele Universitatii "Ovidius" Constanta Seria Matematica, vol. 19, no. 1, pp. 23-34, 2011. [On line]. Available: https://bit.ly/2z5V7kM

[14] S. E. Atani, S. D. P. Hesari, and M. Khoramdel, "Total graph of a commutative semiring with respect to identity-summand elements", Journal of the Korean Mathematical Society, vol. 51, no. 3, pp. 593-607, May 2014, doi: 10.4134/JKMS.2014.51.3.593 
[15] S. E. Atani, S. D. P. Hesari, and M. Khoramdel, "Total Identity-Summand Graph Of A Commutative Semiring With Respect To A Co-Ideal", Journal of the Korean Mathematical Society, vol. 52, no. 1, pp. 159-176, Jan. 2015, doi: 10.4134/JKMS.2015.52.1.159

[16] S. E. Atani and F. E. K. Saraei, "The total graph of a commutative semiring", Analele Universitatii "Ovidius" Constanta - Seria Matematica, vol. 21, no. 2, pp. 21-33, Jun. 2013, doi: 10.2478/auom-2013-0021

[17] S. E. Atani and Z. E. Sarvandi, "The total graph of a commutative semiring with respect to proper ideals", Journal of algebra and related topics, vol. 3, no. 2, pp. 27-41, 2015. [On line]. Available: https://bit.ly/3bM6X0E

[18] I. Beck, "Coloring of commutative rings",Journal of algebra, vol. 116, no. 1, pp. 208-226, Jul. 1988, doi: 10.1016/0021-8693(88)90202-5

[19] B. Bollobas, Graph theory an introductory course. New York, NY: Springer, 1979, doi: 10.1007/978-1-4612-9967-7

[20] J. S. Golan, Semirings and their applications. Dordrecht: Springer, 1999, doi: 10.1007/978-94-015-9333-5

[21] J. Goswami, K. K. Rajkhowa, and H. K. Saikia, "Total graph of a module with respect to singular submodule", Arab journal of mathematical sciences, vol. 22, pp. 242-249, 2016. [On line]. Available: https://bit.ly/3gbI5mu

[22] M. H. Shekarriz, M. H. S. Haghighi, and H. Sharif, "On the total graph of a finite commutative ring", Communications in algebra, vol. 40, no. 8, pp. 2798-2807, Aug. 2012, doi: 10.1080/00927872.2011.585680

[23] Y. Talebi and A. Darzi, "The generalized total graph of a commutative semiring", Ricerche di matematica, vol. 66, no. 2, pp. 579-589, Feb. 2017, doi: 10.1007/s11587-017-0321-4

[24] H. S. Vandiver, "Note on a simple type of algebra in which the cancellation law of addition does not hold", Bulletin of the American Mathematical Society, vol. 40, no. 12, pp. 914-921, Dec. 1934, doi: 10.1090/S0002-9904-1934-06003-8 